\title{
sciendo
}

\section{0-YEAR JOURNEY THROUGH BRAIN GLIOMAS: CASE REPORT}

\author{
COSMIN-NICODIM CÎNDEA ${ }^{1}$, ADRIANA SĂCELEANU ${ }^{2}$ \\ 1,2 "Lucian Blaga”" University of Sibiu, ' Sibiu County Clinical Emergency Hospital
}

Keywords: $\quad$ glioma, Abstract: We present the case of a 28-year-old doctor who was diagnosed in 2009 with grade II doctor, function, brain oligodendroglioma. In the next 10 years he underwent 4 surgeries, passing in turn through 3 different tumour, glioblastoma types of cerebral gliomas: grade II oligodendroglioma, grade III oligoastrocytoma and IDH WildType glioblastoma. In the spirit of the surgical resection limited by the preservation of the function, he was able to practice his medical profession until the last months of his life. Life expectancy at the time of diagnosis complied with all the statistical data. Although the etiology, pathophysiology and treatment of cerebral gliomas is still full of unknowns, knowing our limits we can provide a good quality of life for our patients.

\section{INTRODUCTION}

Glioma tumours are the most common brain tumours. As the name suggests, they originate from glial cells or their precursors. Depending on the cell type, the grade assigned by the World Health Organization (WHO) and the location, they undergo certain classifications.(1)

Depending on the cell type, the main gliomas are: astrocytomas - originating from astrocytes, oligodendrogliomas originating from oligodentrocytes, ependymomas originating from ependymal cells or mixed tumours, such as oligoastrocytomas, which contain mixed cell lines.

According to the grade assigned by the $\mathrm{WHO}$, they are divided into Low-grade (WHO grade II) and High-grade (WHO grade III-IV) gliomas. The low-grade ones are generally well differentiated, with a better prognosis, but still because they have a certain rate of recurrence over time they are considered malignant. The high-grade ones are undifferentiated and have a very unfavourable prognosis.(2)

After localization, we have supratentorial and infratentorial gliomas. The supratentorial ones are more common in adults, and the infratentorial ones are common in children.

\section{CASE REPORT}

A 28-year-old young man, young doctor, was presented at the hospital in 2009 accusing headache and motor aphasia. The cerebral MRI scan showed a left temporal lobe tumour.

After surgery and the anatomopathological result: diagnosis of Oligodendroglioma Grade 2 (low-grade). Life expectancy of patients diagnosed with oligodendroglioma grade 2 , in the high ranked neurosurgical centers, is of 12 years.(3)

In Low-Grade oligodendrogliomas radiotherapy is not useful and chemotherapy is not mandatory (4), the patient chooses to continue without these therapeutic alternatives by performing periodic imaging controls.

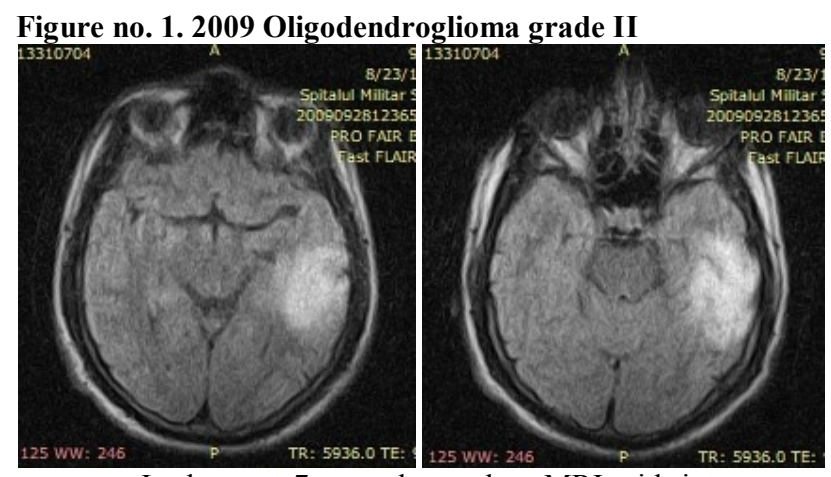

In the next 7 years he made a MRI with intravenous contrast every 12 months to check the postoperative status. No tumour recurrence and no neurological symptoms. He continued to practice his job without impairment.

In 2016 the symptoms recurred and MRI showed a tumour recurrence.

Figure no. 2. 2016 oligoastrocytoma grade III (2007 WHO Classification)

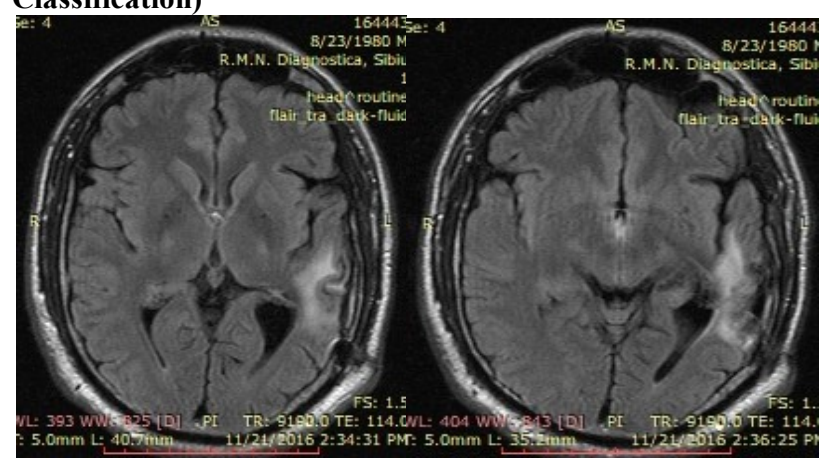

He was operated again and histologically 2 biphasic tumours of the oligodendrolyal and astrocytar type were

${ }^{1}$ Corresponding author: Adriana Săceleanu, B-dul. Corneliu Coposu, Nr. 2-4, Sibiu, România, E-mail: vicentiu.săceleanu@gmail.com, Phone: +40740 022931

Article received on 21.07.2020 and accepted for publication on 24.08.2020 


\section{CLINICAL ASPECTS}

identified, the diagnosis being grade III Oligoastrocytoma (based on 2007 WHO classification).(5)

Postoperatively, the symptomatology was remitted. He was subjected to radiotherapy and chemotherapy. Although the introduction of temozolomide in radiation treatment was an important advance, in recent studies different agents and study combinations have been used for the recurrent and progressive supratentorial treatment of high-grade astrocytomas, the results have been quite disappointing. Response rates are low and progression time is short, ranging from a few weeks to a few months.(6)

Statistically, at the diagnosis time, the life expectancy in the case of grade III oligoastrocitoma is 3.5 years.(7)

Figure no. 3. 2017 check-up cerebral MRI with intravenous contrast

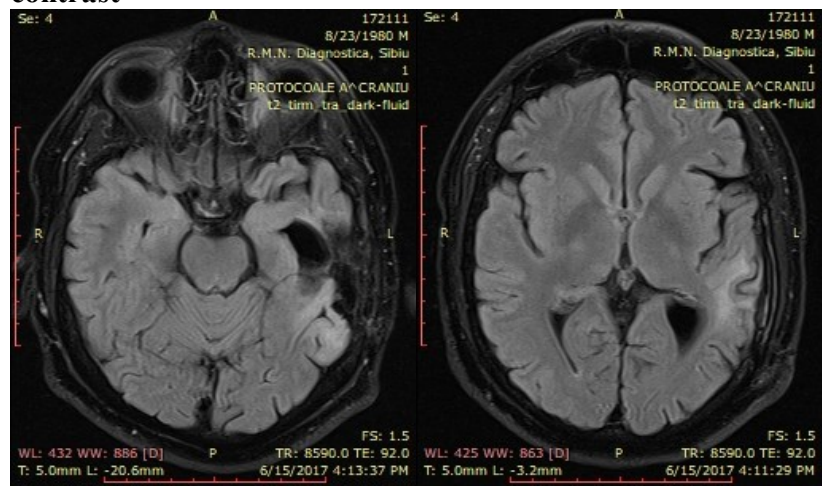

In 2017, the check-up cerebral MRI with intravenous contrast revealed only cerebral gliosis, without tumorous areas. No neurological symptoms. He continued to practice medicine.

In 2018, following the cerebral MRI exam, a tumour recurrence was reported.

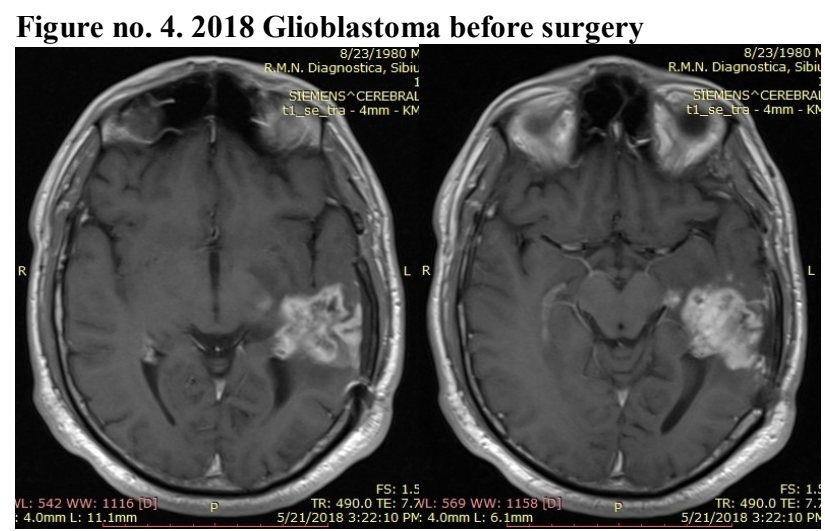

A surgical intervention took place and the diagnosis of

IDH wild-type glioblastoma was established at the histopathological examination and genetic testing for the first time. Life expectancy in IDH Wild-type Glioblastoma is 11-13 months.(8) Surprisingly, IDH Wild-Type GBM is described in the literature as a primary tumour, which develops de novo, without precursor.

In 2019 , he returned with symptoms of aggravated headache, vertigo, motor aphasia and right hemiparesis. The MRI exam confirmed a huge tumour recurrence, with extension in basal ganglia, cerebral peduncles and mesencephalon.

Surgery intervention was done with a subtotal ablation of the tumour, to preserve motor functions, at the patient's choice.

He was discharged with slightly improved neurological functions at:

- 10 years and 3 months from his first surgery (oligodendroglioma grade II);

- 3 years and 3 months from second surgery (oligoastrocytoma grade III);

- 1 year from his third one (glioblastoma grade 4), leading his struggle for survival, and ours, to push the limits of medical knowledge further.

Figure no. 5. 2018 Glioblastoma after surgery

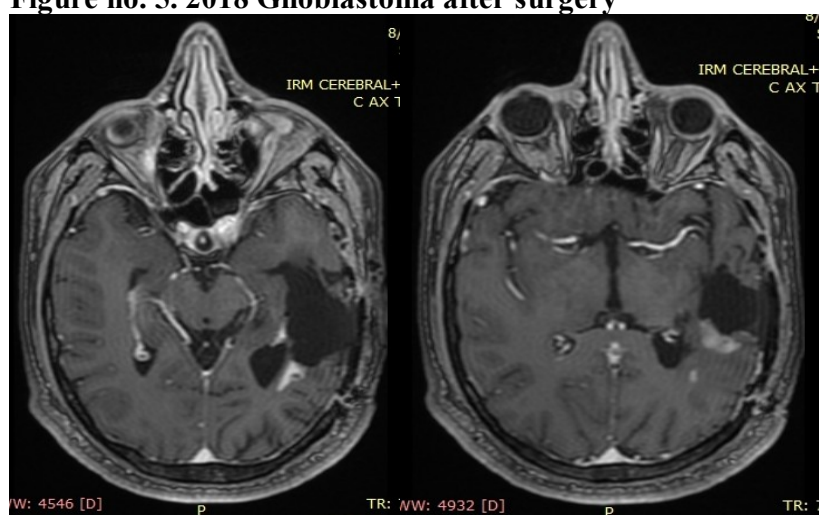

Figure no. 6. 2019 Glioblastoma before surgery

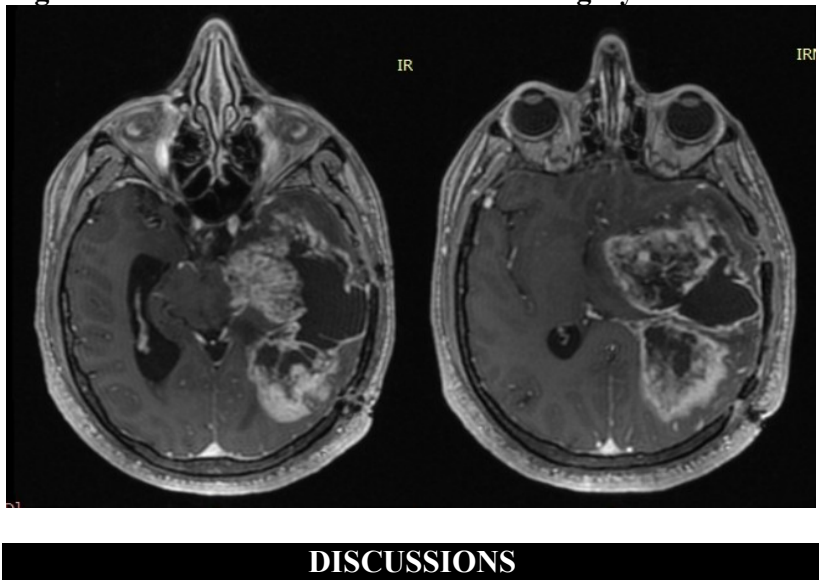

The cause of gliomas and other types of brain tumours is unknown. Cerebral gliomas cannot be prevented.(9)

Abnormalities have been identified in the genes of certain chromosomes that may play a role in tumour development. However, what causes these anomalies is still uncertain. Scientists conduct environmental, occupational, family, and genetic research to identify common links between patients. Despite extensive research into environmental hazards, no direct causes have been found. Most brain tumours are not hereditary. Only $5 \%$ of brain tumours can occur in a genetically inherited context, such as Neurofibromatosis, Li-Frameni, Von HippelLindau, Turcot and Tuberculous Sclerosis.(10)

The current strategy for the treatment of low-grade gliomas is a dynamic one, which challenges the traditional approach by proposing early therapy, repeating treatments and reversing the classical order of therapies (e.g., neoadjuvant chemotherapy when maximum resection is impossible, not early radiotherapy) to improve the quality of life. This philosophy supports a personalized, functional and preventive neurooncology.

Through repeated surgeries, which always followed the principle of limited resection to preserve function, although at the last surgery much of the left cerebral hemisphere was invaded by tumour, the patient had functional speech. This proves that speech function is not limited to the eloquent areas of the dominant hemisphere, but may have an interhemispheric contribution.(11) 


\section{CLINICAL ASPECTS}

In the case of our patient we can discuss certain questions, namely: was the first tumour formation oligodendroglioma grade II cured after tumour resection? We can discuss considering that for 7 years all MRI checks did not show any local tumour recurrence. Then, in the histopathological result, two distinct tumour lines were identified, of oligodendrocytes and astrocytes, making the diagnosis at that time, according to the WHO 2006 classification, of Oligoastrocytoma grade 3. After the WHO 2016 classification we can discuss the coexistence of two tumours, grade 3 astrocytoma and oligodendroglioma.

In 2016, WHO changed the classification based on the genetic testing. No more oligoastrocitoma, can be oligodendroclioma, astrocytoma or both.(12)

Oligoastrocytoma, NOS is an uncommon neuropathology tumour that is no longer a distinct entity. After IDH- $1 / 2$ mutation screening and $\mathrm{LOH} 1 \mathrm{p} / 19 \mathrm{q}$ analysis almost all tumors are either classified as oligodendroglioma or astrocytoma; Oligodendroglioma-like cells: MAP2 +ve (cytoplasm), Astrocytoma-like cells: GFAP +ve (cytoplasm, nuclear membrane).

Another interesting aspect to discuss is that Glioblastoma IDH - wild-type is described in the literature primarily as a primary brain tumour. Mutant IDH glioblastoma is the one that occurs most frequently by escalation from a lower grade glioma.(13) This may raise questions about the accuracy of the genetic test or the impact of radiation therapy on the etiology and development of wild-type glioblastoma. We already know from Prof. Hugues Duffau that the use of radiotherapy in low-grade gliomas is totally contraindicated.

Another important role in the pathophysiology of cerebral gliomas has recently been identified in the metabolism of thrombin generation. In patients with cerebral gliomas, high thrombin generation values were identified. This was also identified in the case of the patient in this case presentation. The use of thrombin inhibitors in the treatment of cerebral gliomas may be attempted in the future.(14) Research in the field of medication delivered through nanoparticles should be followed, which can have important results in the future treatment of this pathology.(15)

\section{CONCLUSIONS}

Surgical treatment is far from having curative claims in brain gliomas, even in the case of very early discoveries. Through surgery we aim to obtain a tumour biopsy for histopathological analysis, cytoreduction of tumour mass with reduction of symptoms caused by the presence of tumour. The decrease in tumour volume is also important for the degree of disease progression. Less remaining tumour tissue will have a lower progression.

Optimally, the neurosurgeon wants to completely excise the tumour, even supramarginal. However, due to the location of the tumour, some tumours cannot be completely removed because this would be done at the cost of significant neurological deficits.

In the multitude of current research, medical experts try all possible approaches: radiotherapy, various combinations of chemotherapy, gene therapy, immunotherapy, stem cells, biomarkers, but looking at the whole picture it seems that we are in a chaotic stage. However, a valuable resource that we can rely on and has been confirmed once again by the case presented, are the statistical data of this pathology.

In the present case, a young doctor honourably practiced his entire medical activity with a cerebral glioma in his head. This proves to us that even with the current reduced therapeutic means, with a good management of the case we can offer beautiful years of life to our patients.

\section{REFERENCES}

1. Wesseling P, Capper D. WHO 2016 Classification of gliomas. Neuropathology and Applied Neurobiology. 2018;44:139-150.

2. Louis DN, Perry A, Reifenberger G, et al. The 2016 World Health Organization Classification of Tumors of the Central Nervous System: a summary. Acta Neuropathol. 2016;131(6):803-820.

3. Claus EB, Walsh KM, Wiencke JK, et al. Survival and low-grade glioma: the emergence of genetic information. Neurosurg Focus. 2015;38(1):E6.

4. Duffau H, Taillandier L. New concepts in the management of diffuse low-grade glioma: Proposal of a multistage and individualized therapeutic approach. Neuro Oncol. 2015; 17(3):332-342

5. Louis DN, Ohgaki H, Wiestler OD, et al. The 2007 WHO classification of tumours of the central nervous system [published correction appears in Acta Neuropathol. 2007 Nov;114(5):547]. Acta Neuropathol. 2007;114(2):97-109.

6. Merrell, R. T., Quant, E. C., \&amp; Wen, P. Y. (2010). Advances in Treatment Options for High-grade GliomaCurrent Status and Future Perspectives. US Neurology, 06(01), 55

7. Walid MS. Prognostic factors for long-term survival after glioblastoma. Perm J. 2008;12(4):45-48.

8. Tamimi AF, Juweid M. Epidemiology and Outcome of Glioblastoma. In: De Vleeschouwer S, editor. Glioblastoma. Brisbane (AU): Codon Publications; 2017 Sep 27. Chapter 8.

9. Săceleanu V. Neurochirurgie Clinică, Edit. ULBS Sibiu; 2014.

10. Hanif F, Muzaffar K, Perveen K, Malhi SM, Simjee ShU. Glioblastoma Multiforme: A Review of its Epidemiology and Pathogenesis through Clinical Presentation and Treatment. Asian Pac J Cancer Prev. 2017;18(1):3-9.

11. Gîță IE. Implications for language acquisition theories: a fresh look at the "genie case". Revista Transilvania. 2016;12:57-60.

12. Kobyakov GL, Absalyamova OV, Poddubskiy AA, Lodygina KS, Kobyakova EA. Klassifikatsiia VOZ pervichnykh opukholeǐ tsentral'nŏ nervnoĭ sistemy 2016 g.: vzgliad klinitsista [The 2016 WHO classification of primary central nervous system tumors: a clinician's view]. Zh Vopr Neirokhir Im N N Burdenko. 2018;82(3):88-96.

13. Huang J, Yu J, Tu L, Huang N, Li H, Luo Y. Isocitrate Dehydrogenase Mutations in Glioma: From Basic Discovery to Therapeutics Development. Front Oncol. 2019;9:506.

14. Cindea C, Mihaila R, Saceleanu, V. Thrombosis Generation Abnormalities In Spontaneous Intracerebral Haemorrhage. Romanian Neurosurgery. 2009;33.

15. Cavalu S, et al. Eco-friendly, Facile and Rapid Way for Synthesis of Selenium Nanoparticles production, structural and morphological characterisation. Revista de Chimie. 2017;68(12):2963-2966. 